\title{
Expedited Discharge of Patients with Diabetic Ketoacidosis in an Emergency Department Short Stay Unit
}

\author{
Parampil Thulasidharan Shibu Kandalam1, Salem Abo Salah1, Peter Alistair Cameron², \\ Jignesh Dhirajlal Trivedi', Muhammed Kunhi Kayakool', Shahsad Ramachan Parambath', \\ Pradeep Radhakrishnan', Joe Varghese Mathew ${ }^{1}$, Jaseem Sirajudeen ${ }^{1}$ \\ ${ }^{1}$ Department of General Internal Medicine, Hamad General Hospital, Doha, Qatar \\ ${ }^{2}$ Department of Epidemiology \& Preventive Medicine, Monash University, Melbourne, Australia \\ Email: shibudoc@yahoo.co.in, pkandalam@hamad.qa, jtrivedi@hamad.qa,sparambath@hamad.qa, jsirajudeen@hamad.qa
}

How to cite this paper: Kandalam, P.T.S., Salah, S.A., Cameron, P.A., Trivedi, J.D., Kayakool, M.K., Parambath, S.R., Radhakrishnan, P., Mathew, J.V. and Sirajudeen, J. (2019) Expedited Discharge of Patients with Diabetic Ketoacidosis in an Emergency Department Short Stay Unit. Open Journal of Internal Medicine, 9, 112-120. https://doi.org/10.4236/ojim.2019.94016

Received: October 1, 2019

Accepted: November 3, 2019

Published: November 6, 2019

Copyright $\odot 2019$ by author(s) and Scientific Research Publishing Inc. This work is licensed under the Creative Commons Attribution International License (CC BY 4.0).

http://creativecommons.org/licenses/by/4.0/

\section{(c) (i) Open Access}

\begin{abstract}
Aim: Review of presentation, management and outcome of patients admitted with Diabetic Ketoacidosis (DKA) to an emergency department short stay unit with expedited discharge. Methods: All admitted patients with a discharge diagnosis of "Diabetic Ketoacidosis", were identified by the file auditing section in the Emergency Department. Data obtained from the medical records were collected using an explicit chart review from January 2012 to June 2013. Data included clinical monitoring, investigations performed, the type and amount of intravenous fluids given, the insulin regime, potassium supplementation and outcome. Results: Out of a total of 120 patients labelled as DKA or hyperglycaemia on arrival, hundred patients fulfilled the criteria for DKA. In the population studied the mean age was 25 years with a male predominance. Eighty-two patients suffered from Type 1 Diabetes Mellitus (T1DM) whilst eighteen patients had Type 2 Diabetes Mellitus (T2DM). Sixteen patients were newly diagnosed during the present admission. Seventy-six (76\%) patients were on insulin. The insulin regimen and potassium supplementation were followed as per protocol in all patients. All the patients except one were given intravenous fluid according to protocol. Parameters were monitored adequately except fluid input and output monitoring. The median length of stay in the short stay unit was 1.5833 days. There was no return visit within one week of discharge. Conclusion: The median length of stay was short and there were no documented complications or deaths during the stay. There was poor compliance with documentation of fluid input and output. In this population, the short stay model of care appeared to be safe and efficient.
\end{abstract}




\section{Keywords}

Diabetes Mellitus, Diabetic Ketoacidosis, DKA Audit, Emergency

Department, Expedited Discharge, Length of Stay

\section{Introduction}

Diabetic Ketoacidosis (DKA) is an uncontrolled state of catabolism associated with insulin deficiency, excess of counter regulatory hormone and fluid depletion. It is the hallmark of T1DM and can be the mode of presentation. Patients with T2DM are also at risk of developing DKA as well especially during the catabolic stress of acute illness [1] [2]. DKA adds to morbidity and mortality amongst patients with diabetes. Mortality rates in DKA, quoted at $<5 \%$, increase substantially with increasing age and concomitant critical illnesses [1]-[6].

While treating patients with DKA, the points to be considered and closely monitored are: Correction of fluid loss with intravenous fluids, controlled reduction of hyperglycaemia with insulin, balancing of electrolyte, particularly potassium, return of acid-base disturbance and addressing potential concurrent infection. Fluid resuscitation dilutes both the blood glucose and that of circulating counter-regulatory hormones. Insulin is needed to stop cata-bolic state and resume uptake of glucose in tissues, checking gluconeogenesis and free fatty acid generation and hence ketone production.

It is weight-based fixed rate intravenous insulin infusion that is now recommended than bolus dose of insulin delivery, until ketosis has subsided (The 2013 Joint British Diabetes Societies (JBDS) guideline). The dose of Insulin infusion is at a rate of $0.1 \mathrm{U} / \mathrm{kg} / \mathrm{h}$ [3]. The JBDS advises admission to a Level 2 high-dependency unit when DKA is severe with any of the following features 1) blood ketones more than $6 \mathrm{mmol} / \mathrm{l}$; 2) bicarbonate lower than $5 \mathrm{mmol} / \mathrm{l}$; 3) venous $\mathrm{pH}$ below 7.1 ; 4) significant hypokalemia on admission (below $3.5 \mathrm{mmol} / \mathrm{l}$ ); 5) Low Glasgow Coma Scale (less than 12); 6) hypoxia $\left(\mathrm{SpO}_{2}\right.$ below $92 \%$ breathing room air); 7) blood pressure below $90 \mathrm{mmHg}$ systolic; 8) tachycardia over 100 or bradycardia below 60 per min and 9) anion gap more than 16 [anion gap $=\left(\mathrm{Na}^{+}+\right.$ $\left.\left.\mathrm{K}^{+}\right)-\left(\mathrm{Cl}^{-}+\mathrm{HCO}_{3}^{-}\right)\right][3]$.

The aim of this audit was to review the presentation, management and outcome of patients admitted with DKA to the emergency department and to assess safety and efficiency of patient care in an emergency department short stay observation unit.

\section{Method}

\subsection{Setting}

At Hamad General Hospital (HGH) Emergency Department (ED) in Qatar, there are approximately $1300-1500$ adult attendances per day. It is the major tertiary hospital for a country of approximately 2.5 million people, with a skewed 
population including a large percentage of single male labourers less than 40 years of age.

In $\mathrm{HGH}$, there is a designated short stay unit (SSU) as a part of ED with an admission time frame of less than 72 hours to manage and discharge acute medical cases. The ED short stay unit is managed by a team of internal medicine and emergency medicine trained consultants and specialists with 24 hour cover and it manages 600 to 800 medical cases per month.

\subsection{Diagnosis}

Patients admitted to the ED in HGH were diagnosed as DKA if they fulfilled the following criteria, as per DKA protocol (issued in 2008 available on the HMC intranet site): 1) hyperglycaemia, 2) ketonemia or ketonuria, and 3) serum bicarbonate less than $18 \mathrm{mEq} / \mathrm{L}$ or venous $\mathrm{pH}$ less than 7.3 (high anion gap metabolic acidosis). This protocol provided guidelines on monitoring, investigations, therapeutic intervention and disposition. Instructions on fluid balance, insulin infusion rates and potassium supplements were given. The protocol also mentioned indications for sodium bicarbonate use. The protocol used in HGH is based on JBDS and American diabetic association recommendations.

\subsection{Patients}

Inclusion criteria: The patients studied were adult patients ( $\geq$ fourteen years of age) presenting to the emergency department, HGH with "DKA" between January 2012 and June 2013 as per admission records in the ED.

Exclusion criteria: Patients with other significant clinical conditions causing the presentation, such as stroke, were admitted to respective inpatient units and were not included in this review.

Data were extracted using an explicit chart review from patient medical records according to the Data Protection Act. Data were collected and information regarding the parameters monitored was entered using the proforma. The following parameters were monitored-age, sex, reason for admission, prior insulin prescription, IV insulin initiated, IV fluid type and amount, potassium supplementation, changing IV to subcutaneous insulin, monitoring (pulse, blood pressure, temperature, fluid intake and output), diabetic education and readmission within one week of discharge.

Eleven cases were admitted to Medical Intensive Care Unit (MICU) as per MICU admission criteria (bicarbonate lower than $5 \mathrm{mmol} / \mathrm{l}$, venous or arterial $\mathrm{pH}$ lower than 7.1, GCS less than 12, persistent low blood pressure below 90 $\mathrm{mmHg}$ (systolic) after initial four hour fluid resuscitation) and treated according to the coexisting medical conditions. Three out of this eleven patients were stepped down to the short stay unit in twenty four hours and the remaining eight patients were later on shifted to the medical ward and discharged home from there.

Quantitative data (age, length of stay) were collected and analysed using de- 
scriptive statistical methods. Table 1 shows the demographic and clinical characteristics of patients.

The project was approved as a quality improvement project by the Hamad Medical Corporation (HMC) IRB \& Medical Research Centre (MRC).

\section{Results}

Out of a total of 120 patients labelled as DKA or hyperglycemia one hundred patients fulfilled the criteria for DKA. In this population the mean age (Figure 1) was 25 years with a male predominance $(n=59)$. Eighty two patients suffered from T1DM, eighteen patients suffered from T2DM. Eighty four patients were known diabetic and sixteen were newly diagnosed during the presentation to ED.

The median length of stay (LOS) in short stay unit was 1.5833 days [38 hours]. Out of the eighty two patients with T1DM, seventy four patients were on a fixed insulin regime whilst seven were newly diagnosed and one was not on medication.

Table 1. Demographic and clinical characteristics of patients.

\begin{tabular}{|c|c|}
\hline Characteristics & $\begin{array}{l}\text { All patients } \\
\qquad N=100\end{array}$ \\
\hline Age: mean in years & $25[\mathrm{SD}=11.99]$ \\
\hline Range: years & $14-72$ \\
\hline Male \% & 59 \\
\hline Female $\%$ & 41 \\
\hline Type 1 DM \% & 82 \\
\hline Type 2 DM \% & 18 \\
\hline Number of patients on Insulin & 76 \\
\hline Number of patients newly diagnosed & 16 \\
\hline \multicolumn{2}{|l|}{ Reason for DKA } \\
\hline Noncompliance to Insulin regimen & 61 \\
\hline Infection/Febrile illness & 11 \\
\hline Others-no reason found & 28 \\
\hline \multicolumn{2}{|l|}{ Investigations performed } \\
\hline Blood gas. \% & 89 \\
\hline Urea, electrolytes and ketones. \% & 100 \\
\hline Investigations find source of infection. \% & 100 \\
\hline \multicolumn{2}{|l|}{ Disposition } \\
\hline Short stay unit admission & 89 \\
\hline MICU admission & 8 \\
\hline $\begin{array}{l}\text { Medical ward admission } \\
\text { Length of hospital stay (LOS) }\end{array}$ & 3 \\
\hline Median LOS in short stay unit & 1.5833 days $[38 \mathrm{hrs}]$ \\
\hline
\end{tabular}




\section{Age distribution of patients}

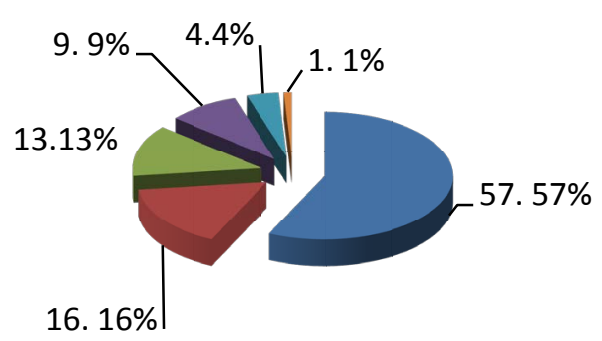

14-20yrs

21-30yrs

31-40yrs

41-50yrs

51-60yrs

$61-80 y r s$

Figure 1. Age distribution of patients with DKA at presentation.

Three of the T2DM patients were on insulin whilst three were on oral hypoglycaemic agents. One patient was on controlled diabetic diet only and the others were newly diagnosed.

\subsection{Precipitating Factors}

Noncompliance with insulin regimen was documented in sixty one out of seventy six patients on insulin. Eleven patients had infective or febrile illness at the time of presentation, one had a gluteal abscess, seven had upper respiratory tract infection, one had urinary tract infection, one had anacute febrile illness and one patient had sepsis of unknown origin (Figure 2).

\subsection{Investigations}

All except two had a stat blood sugar at the time of presentation. Two patients did not have their random blood sugar (RBS) checked because they were not known to be diabetic before, whilst fourteen others without a past history of diabetes had their initial blood sugar because presentation suggested DKA. All the patients had temperatures recorded and complete blood count, renal function and electrolytes checked at the time of admission. Serum ketones were checked in all patients including the patients who were previously not known to be diabetic. Eighty nine (89\%) had an ABG or VBG at the time of admission. The majority had relevant investigations done for anysource of infection.

\subsection{Venous Blood Gases (VBGs)}

According to the DKA protocol VBG is preferred over ABG and it is mandatory in the setting of hypotension, mental status change or critical illness. However only eighty nine (89\%) patients had blood gases and this was mostly an ABG.

\subsection{Serial Parameter Monitoring}

The current protocol mandates hourly glucose monitoring, fluid input/output charting, mental status documentation and 2 - 4 hourly plasma electrolytes. Urea and electrolytes were checked in all patients but it was repeated only at a six to 


\section{Precipitating event}

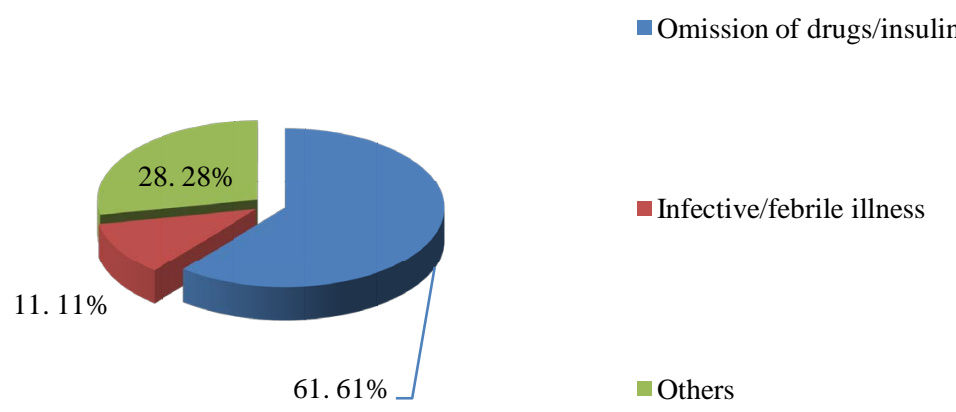

Figure 2. Major precipitating events in percentage.

eight hour interval for most of the patients. Documentation of mental status and hourly vital signs only occurred on admission and while patient stayed in the resuscitation room. In the short stay unit it was recorded every second hour. Input/output monitoring was poorly completed (45\%).

\subsection{Insulin Administration}

Intravenous insulin infusions were administered in all the patients. Hourly bedside glucose monitoring was routinely done in all patients and insulin dose was adjusted according to the sliding scale. There was no documentation regarding any complication of treatment. All the patients were changed to subcutaneous insulin with 1 - 2 hours overlap of intravenous and subcutaneous doses.

\subsection{Intravenous Fluid Administration}

All the patients except one achieved the goal of 1 - 2 litres of fluid in the first hour. One patient was diagnosed as DKA only after two hours because the clinical presentation was not suggestive. The patients had a change of IV fluid from normal saline to dextrose. We did not observe data regarding calculation of corrected sodium and changing normal saline to $0.45 \%$ saline in any of the records. But few patients received $0.45 \%$ saline.

\subsection{Potassium Administration}

All the patients were started on a potassium infusion which was consistent with the protocol. Some of the patients received a lesser dose considering the plasma potassium value.

\subsection{Sodium Bicarbonate $\left(\mathrm{NaHCO}_{3}\right)$}

Only two patients were administered $\mathrm{NaHCO}_{3}$ in view of a deteriorating $\mathrm{pH}$ of $<7$ despite administering adequate amounts of insulin and fluids.

\subsection{Disposition}

Out of hundred patients, eighty six patients were directly admitted to the short 
stay unit. Three patients needed to be admitted to the medical floor and two were discharged after six days and one was discharged after three days. Eleven patients were admitted to MICU but three were stepped down within twenty four hours to the short stay unit (included in the study). Eight patients were continued in MICU and later on shifted to the medical ward (Figure 3).

\subsection{Length of Hospital Stay}

The duration of stay of those eighty nine patients admitted to the short stay unit is shown in Figure 4. The median duration of hospitalization for patients admitted to the short stay unit was 1.5833 days [ 38 hours]. Figure 5 shows the length of stay of patients admitted to MICU and Medical ward.

\section{Discussion}

In our retrospective study we analysed clinical presentation, management and outcome of DKA patients in an emergency short stay unit.

The median length of stay of patients admitted to short stay unit analysed was

\section{Disposition}

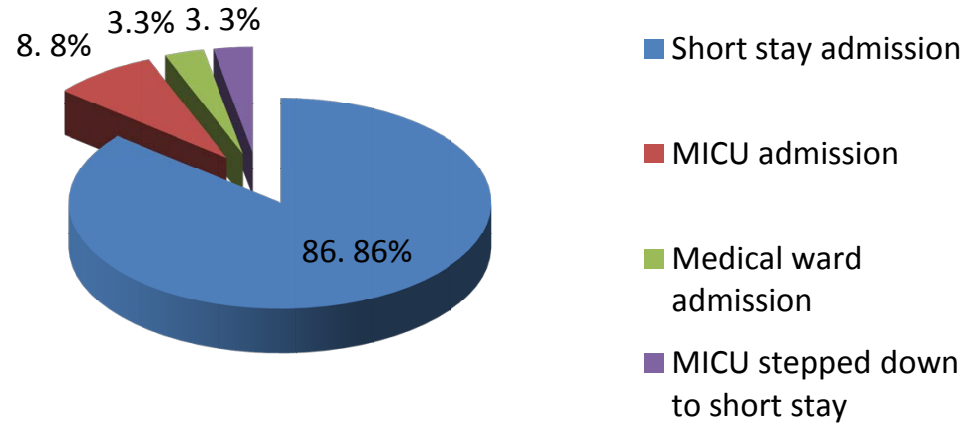

Figure 3. Disposition of patients of DKA from emergency department to various units. 86 out of 100 patients admitted to short stay unit.

\section{Length of stay in short stay unit \\ (Total 89 patients)}

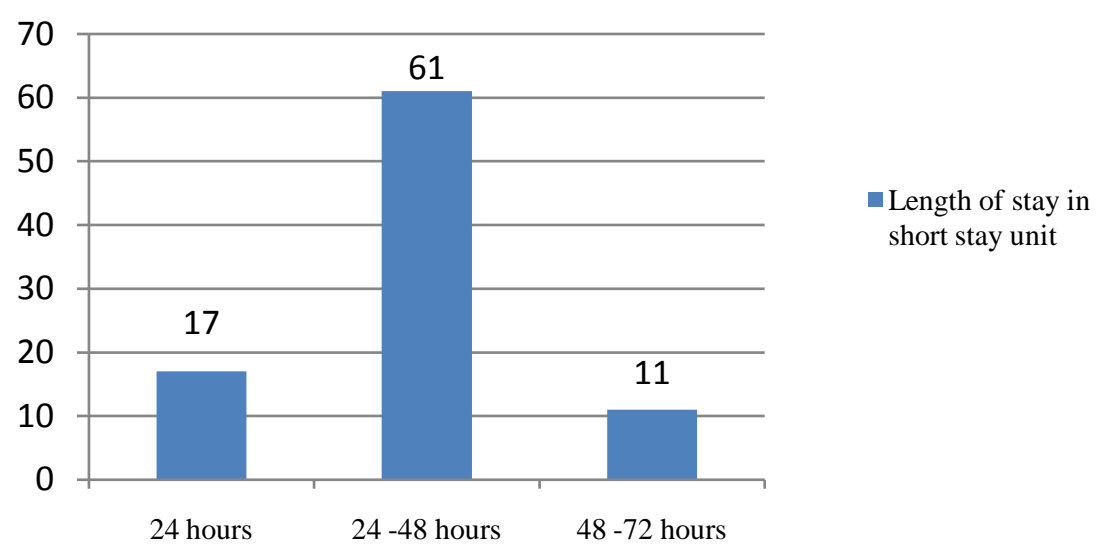

Figure 4. Length of stay of patients admitted to short stay unit in hours. 


\section{Length of hospital stay of patients admitted to MICU and Medical} Ward

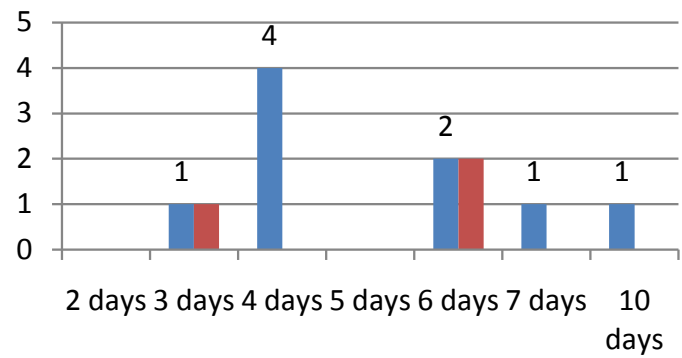

Length of stay of MICU admitted patients

Length of stay of medical ward admitted patients

Figure 5. Length of stay of the patients admitted to MICU and medical ward.

1.5833 days. This is much shorter than that reported in other countries, even when the MICU cases are included [1] [4] [7] [8]. There were no documented adverse consequences associated with the short length of stay.

The emergency department short stay beds are monitored and staffed at a higher level than normal ward staffing. It is likely the safe and efficient management of DKA patients was related to the continuous specialist coverage and well trained nursing staff.

The majority of patients admitted with DKA suffered from T1DM whilst eighteen patients suffered from T2DM.

Noncompliance by patients of their insulin regimen was documented in sixty one out of seventy six patients on insulin. This is in line with many studies done on this subject, accounting for nearly two thirds of cases (except DKA as first presentation of Diabetes Mellitus) [2] [5] [6].

Deviations from the protocol were noted during the patients' stay in the ED. Input and output charting was not requested and not done in many patients. Most of the patients had their blood gases taken more than one time. There was no record of hourly mental status except the routine admission documentation by the nurse and when in the resuscitation room. The frequency of blood gas monitoring is not mentioned in the present protocol, but does not appear necessary for most cases after the initial sample.

All the patients were started on a potassium infusion which was consistent with the protocol. Some of the patients received less potassium than directed by the protocol according to the plasma potassium value.

We achieved a rapid reversal and improvement in abnormal $\mathrm{pH}$ and ketosis by timely diagnosis and early initiation of fluid resuscitation and intravenous insulin therapy [1] [2] [4] [6]. We usually did not change the fluid back to saline once it was changed to dextrose containing fluid unless the blood sugar was not controlled with incremental doses of intravenous insulin.

Many episodes of DKA can be prevented through better patient education. We achieved better patient education through our dedicated patient educator team who provided comprehensive counselling and education to all patients be- 
fore discharge.

In our Hospital the short stay unit of the ED played a major role in managing cases of DKA and avoided a significant number of inpatient admissions with rapid turnaround of critically ill patients. This approach reduced the cost of management as well as improving access block in the hospital.

This project was a retrospective review and has its limitations. Some files were missing or difficult to trace and some case files had insufficient documentation of history regarding omission of medication. Despite this, we identified all admitted cases and were able to track all cases to discharge.

\section{Conclusion}

This review has demonstrated that DKA can be managed safely and efficiently in a short stay observation unit [1] [7] [8].

\section{Conflicts of Interest}

The authors declare no conflicts of interest regarding the publication of this paper.

\section{References}

[1] Abela, A.-G., Magri, C.J., Debono, M., Calleja, N., Vassallo, J. and Azzopardi, J. (2008) An Audit of the Management of Diabetic Ketoacidosis at St Luke's Hospital. Malta Medical Journal, 20, 16-21.

[2] Kitabchi, A.E., Umpierrez, G.E., Murphy, M.B., Barrett, E.J., Kreisberg, R.A., Malone, J.I., et al. (2001) Management of Hyperglycaemic Crises in Patients with Diabetes. Diabetes Care, 24, 131-153. https://doi.org/10.2337/diacare.24.1.131

[3] Savage, M.W., Dhatariya, K.K., Kilvert, A., Rayman, G., Rees, J.A.E., Courtney, C.H., Hilton, L., Dyer, P.H. and Hamersley, M.S. (2011) Joint British Diabetes Societies Guideline for the Management of Diabetic Ketoacidosis. Diabetic Medicine, 28, 508-515. https://doi.org/10.1111/j.1464-5491.2011.03246.x

[4] Dawkins, C.L. and Oyibo, S. (2012) The Management of DKA in Busy District Hospital, Department of Diabetes \& Endocrinology, Peterborough City Hospital, Bretton Gate, Peterborough, PE39GZ, UK. The Online Journal of Clinical Audits, 4. https://www.researchgate.net/publication/335160346

[5] Umpierrez, G.E., Murphy, M.B. and Kitabchi, A.E. (2002) Diabetic Ketoacidosis and Hyperglycaemic Hyperosmolar Syndrome. Diabetes Spectrum, 15, 28-36. https://doi.org/10.2337/diaspect.15.1.28

[6] Hardern, R.D. and Quin, N.D. (2003) Emergency Management of Diabetic Ketoacidosis in Adults. Emergency Medical Journal, 20, 210-213.

https://doi.org/10.1136/emj.20.3.210

[7] Freire, A.X., Umpierrez, G.E., Afessa, B., Latif, K.A., Bridges, L. and Kitabchi, A.E. (2002) Predictors of Intensive Care Unit and Hospital Length of Stay in Diabetic Ketoacidosis. Journal of Critical Care, 17, 207-211.

[8] Paul, A., Fowler, L., Reddy, R. and Elsheikh, M. (2004) Audit of Management of Diabetic Ketoacidosis in a Busy District General Hospital. Endocrine Abstracts, 8, DP8. 